\title{
Reconstruction and analysis of genome-scale metabolic model of a photosynthetic bacterium
}

\author{
Arnau Montagud ${ }^{1,3^{*}}$, Emilio Navarro², Pedro Fernández de Córdoba ${ }^{1}$, Javier F Urchueguía', Kiran Raosaheb Patil ${ }^{3}$
}

\begin{abstract}
Background: Synechocystis sp. PCC6803 is a cyanobacterium considered as a candidate photo-biological production platform - an attractive cell factory capable of using $\mathrm{CO}_{2}$ and light as carbon and energy source, respectively. In order to enable efficient use of metabolic potential of Synechocystis sp. PCC6803, it is of importance to develop tools for uncovering stoichiometric and regulatory principles in the Synechocystis metabolic network.

Results: We report the most comprehensive metabolic model of Synechocystis sp. PCC6803 available, iSyn669, which includes 882 reactions, associated with 669 genes, and 790 metabolites. The model includes a detailed biomass equation which encompasses elementary building blocks that are needed for cell growth, as well as a detailed stoichiometric representation of photosynthesis. We demonstrate applicability of isyn669 for stoichiometric analysis by simulating three physiologically relevant growth conditions of Synechocystis sp. PCC6803, and through in silico metabolic engineering simulations that allowed identification of a set of gene knock-out candidates towards enhanced succinate production. Gene essentiality and hydrogen production potential have also been assessed. Furthermore, iSyn669 was used as a transcriptomic data integration scaffold and thereby we found metabolic hot-spots around which gene regulation is dominant during light-shifting growth regimes.
\end{abstract}

Conclusions: iSyn669 provides a platform for facilitating the development of cyanobacteria as microbial cell factories.

\section{Background}

Cyanobacteria, which have been model organisms since the early 70s of the past century [1], are a widespread group of photoautotrophic microorganisms, which originated, evolved, and diversified early in Earth's history [2]. It is commonly accepted that cyanobacteria played a crucial role in the Precambrian phase by contributing oxygen to the atmosphere [3]. All cyanobacteria combine the ability to perform an oxygenic photosynthesis (resembling that of chloroplasts) with typical prokaryotic features, like performing anoxygenic photosynthesis by using hydrogen sulfide $\left(\mathrm{H}_{2} \mathrm{~S}\right)$ as the electron donor or fixing atmospheric dinitrogen $\left(\mathrm{N}_{2}\right)$ into ammonia $\left(\mathrm{NH}_{3}\right)$. Relevance of this phylum covers from evolutionary studies [4] to biotechnological applications, including biofuel production [5]. Synechocystis sp. PCC6803 is a cyanobacterium that is considered as a good candidate

\footnotetext{
* Correspondence: armontag@mat.upv.es

${ }^{1}$ Instituto Universitario de Matemática Pura y Aplicada, Universidad

Politécnica de Valencia, Camino de Vera 14, 46022 Valencia, Spain

Full list of author information is available at the end of the article
}

for developing a photo-biological cell factory towards production of a variety of molecules of socio-economic interest, with $\mathrm{CO}_{2}$ (and/or sugars) as carbon source and light (and/or sugars) as energy source [6]. The diversity of potential applications in this sense is broad. Works have been published on heterologous production of metabolites such as isoprene [6], poly-beta-hydroxybutyrate [7], biofuels [8] and bio-hydrogen [9,10] - an energy vector of global interest [11].

Synechocystis sp. PCC6803 is capable of growing under three different growth conditions as marked by the utilized carbon source (/s) [12]. This causes that three distinct modes of operation are interweaved over the same metabolic network, viz., i) photoautotrophy, where energy comes from light and carbon from $\mathrm{CO}_{2}$; ii) heterotrophy, where energy and carbon source is a saccharide, for instance glucose; and, iii) mixotrophy, a combination of the above two, where light is present as well as a combination of two carbon sources: glucose and $\mathrm{CO}_{2}$. Reconstruction of a genome-scale metabolic model for this model photo-synthetic bacterium is one 
of the main goals of the current study. Genome-scale metabolic network reconstruction is, in essence, a systematic assembly and organization of all the reactions which build up the metabolism of a given organism; and has been of great interest in the post-genomic era. The variety of applications of such a metabolic model [13] includes the possibility of assessing projects for the production and optimization of an added value metabolite. If a model is formulated properly, it is expected to allow simulating environmental and genetic perturbations in the metabolic network. Thus, together with appropriate constraints, a metabolic model would partially represent a virtual organism - an in silico model that allows probing possible flux distributions inside the cell under different environmental conditions and for a given genetic make-up. Towards this end, a variety of tools/algorithms are available [14], including flux balance analysis (FBA) $[15,16]$, minimization of metabolic adjustments (MOMA) [17], regulatory on-off minimization (ROOM) [18] and metabolic control analysis (MCA) [19,20].

Synechocystis sp. PCC6803 genome was sequenced, annotated and made publicly available in 1996 [21,22] and has been the target of some metabolic modeling effort, especially for central carbon metabolic reconstructions [23,24]. The work from Yang et al [23] focused on a metabolic model of glycolysis, tricarboxylic acid cycle and pentose phosphate pathway that was simulated under heterotrophic and mixotrophic conditions. Shastri and Morgan [24] studied a metabolic model with the same pathways under autotrophic conditions and compared their results to the ones from Yang et al. These two works considered one lumped reaction for the photosynthesis of the system. More recently, an uncurated reaction list with a biomass composition represented by central carbon metabolites has been published [25]. This model, however, is not suitable for simulations due to lack of proper biomass equation, lumped nature of some key reactions and missing reactions.

The large quantity of information featured in public databases, like details about genomes [26], pathways [27], enzymes [28] or proteins [29] can be used from different databases to gather all published data for one specific organism. However, the lack of quality must be considered as a major drawback of some of the databases: false positives, false negatives as well as wrongly annotated objects may hinder efforts of collecting accurate data [30]. Consequently, manual reconstruction by detailed inspection of each and every reaction, biomass equation based on metabolic building blocks (such as amino acids and nucleotides), consistency and integrity of the network is a pre-requisite for creating a high quality and useful metabolic model [31]. The current study presents such manually curated reconstruction for
Synechocystis sp. PCC6803 and demonstrates some of its potential applications.

The present model features a detailed biomass equation which encompasses all the building blocks that are needed for a flux distribution simulation that reflects observed phenotype. No lumped reactions are present and photosynthesis is described as a set of 19 reactions, thus enabling the tracing of the corresponding fluxes. Furthermore, different analyses are performed by using this metabolic reconstruction, including reaction knockout simulations, flux variability analysis and identification of transcriptional regulatory hotspots. Overall, $i$ Syn669 is a valuable tool towards the development of a photo-biological production platform. The model will also contribute to the existing set of genome-scale models with a virtue of being one of the first stoichiometric models that account for photosynthesis.

\section{Results and Discussion}

\section{Genome-scale metabolic network reconstruction}

A complete literature examination, including databases, biochemistry textbooks and the annotated genome sequence, was needed in order to extract the current state of the art on known metabolic reactions within the metabolic network of Synechocystis sp. PCC6803. For a thorough overview of the process of metabolic model reconstruction, refer to very instructive work by Forster et al [32] as well as review by Feist et al [31]. In detail, the reconstruction started with the annotation and genomic sequence files of Synechocystis sp. PCC6803 $[21,22]$. These files were used with Pathway Tools software [33] in order to build a database of all the genes, proteins and metabolites presents in the organism. The list of reactions was then retrieved from Pathway Tools; EC numbers and stoichiometry of the reactions were checked and verified with the help of the Enzyme nomenclature database [34] and KEGG pathway database [27]. Reactions were elementally balanced except for protons, so that chemical conversions were coherent. In some of the reactions present in these databases, metabolites were reported in a non-specific form (e.g. 'an alcohol'). This is insufficient for metabolic model simulation and, so, corresponding organism-specific metabolites had to be identified [32]. Additionally, in a large number of reactions cofactors were not completely clarified: an enzyme being capable of using NADH or NADPH or both. In the latter, two reactions were included in the reconstructed metabolic network. Determination of reversibility of the reactions was assisted by specific enzyme databases, like BRENDA [28]. If no conclusive evidence was reported, reactions were set to be reversible.

In the reconstruction of the metabolic model, many reactions (a total of 79 reactions, see Table 1) were 
Table 1 Distribution of the model reactions as per cognate genes

\begin{tabular}{ll}
\hline Number of reactions & 882 \\
\hline With assigned genes & 669 \\
\hline - Protein-mediated transport & 78 \\
\hline C Chemical conversion & 221 \\
\hline Transport reactions & 47 \\
\hline EC reactions not annotated & 20 \\
\hline Needed for biomass simulation & 79 \\
\hline
\end{tabular}

found to be necessary for the production of the monomers, precursors or building blocks, that are considered in the biomass equation but which have no corresponding enzyme coding gene assigned. In consequence, many genes that were not annotated before should be considered, as they code for enzymes that should be present to allow the formation of biomass. For instance, enzymes malyl-CoA lyase and isocitrate lyase were not allocated in the annotation of the genome albeit their activities have been measured $[35,36]$ and their presence is necessary to complete the glyoxylate shunt; consequently, they were included in the model.

The product of this reconstruction process was a set of reactions that encompass all the known metabolite conversions that take place in Synechocystis sp. PCC6803. The resulting network, iSyn669, consists of 882 metabolic reactions and 790 metabolites (see Table 1 for more information). A total of 669 genes were included, to which 639 reactions were assigned (see Additional file 1 for details); the difference between the number of genes and assigned reactions is due to the presence of considerable number of protein complexes (e.g. photosynthetic or respiratory activities) and isoenzymes. Reactions with no cognate genes are also present in $i$ Syn669, 20 passive transport reactions and 47 chemical conversions (not mediated by enzymes) were included. Additionally, a total of 79 reactions were included on the basis of biochemical evidence or physiological considerations, but currently with no annotated Open Reading Frame (ORF). iSyn669 genome-scale metabolic model is available in Additional file 2 (in OptGene [37] format).

iSyn669 spans all the biologically relevant flux nodes in the Synechocystis metabolism. Pyruvate, phosphoenolpyruvate (PEP), 3-phosphoglycerate, erythrose-4-phosphate and 2-oxoglutarate are main flux nodes for amino acids biosynthesis. Acetyl-CoA is an important flux node for fatty acids production, with high relevance for metabolic engineering towards biofuel production. Biosynthesis of nucleic acids comes from different metabolites, namely, ribose-5-phosphate, 5-phospho-beta-D- ribosyl-amine, L-histidine and L-glutamine. Moreover, with the information publicly available on databases, we can conclude that Synechocystis sp. PCC6803 bears an incomplete tricarboxylic acid cycle (TCA cycle), as it lacks 2-ketoglutarate dehydrogenase (EC 1.2.4.2). It has been published that glyoxylate shunt completes this cycle [35], permitting the recycling of TCA metabolites. Alternatively, aspartate transaminase (reaction 2.6.1.1a in iSyn669) can interconvert 2-ketoglutarate and oxaloacetate, thus bridging the gap of 2-ketoglutarate dehydrogenase, but short-circuiting TCA cycle.

From the network topology perspective, iSyn669 displays the connectivity distribution pattern similar to that of the other microbial genome-scale networks, e.g. yeast [32] and Escherichia coli [38] (Table 2). While most of the metabolites have few connections, few metabolites are involved in very many reactions and are often referred to as metabolic hubs. Homeostasis of such highly connected metabolites will affect globally the metabolic phenotype (as reflected in metabolite levels and fluxes) and therefore of interest for studying the organization of regulatory mechanisms on the genomewide scale. Most connected metabolites include those related to energy harvesting (e.g. ATP, NADP+, oxygen), a key metabolite in the porphyrin and chlorophyll metabolism (S-adenosyl methionine), a couple of amino acids

Table 2 Most connected metabolites in the iSyn669 metabolic network

\begin{tabular}{llll}
\hline Metabolite & Neighbors & $\begin{array}{l}\text { Neighbors } \\
\text { in E. coli }\end{array}$ & $\begin{array}{l}\text { Neighbors } \\
\text { in yeast }\end{array}$ \\
\hline H2O & 213 & 697 & - \\
\hline ATP & 144 & 338 & 166 \\
\hline phosphate & 108 & 81 & 113 \\
\hline ADP & 103 & 253 & 131 \\
\hline diphosphate & 97 & 28 & - \\
\hline H+ & 74 & 923 & 188 \\
\hline CO2 & 72 & 53 & 66 \\
\hline NADP+ & 64 & 39 & 61 \\
\hline NADPH & 63 & 66 & 57 \\
\hline NAD+ & 46 & 79 & 58 \\
\hline L-glutamate & 45 & 52 & 56 \\
\hline NADH & 42 & 75 & 52 \\
\hline AMP & 36 & 86 & 48 \\
\hline oxygen O2 & 36 & 40 & 31 \\
\hline ammonia & 28 & 22 & - \\
\hline S-adenosyl-L-methionine & 25 & 18 & 19 \\
\hline glutathione & 25 & 17 & 10 \\
\hline a malonyl-ACP & 23 & 15 & 10 \\
\hline L-glutamine & 22 & 18 & 23 \\
\hline coenzyme A & 21 & 71 & 39 \\
\hline
\end{tabular}


and its precursors (L-glutamate, L-glutamine and glutathione) and a key metabolite in the lipid biosynthesis pathway (malonyl-ACP). High connectivity of these metabolites hints to their potential central role in the re/adjustments of fluxes following environmental changes/perturbations. In order to discover the corresponding regulatory mechanisms, additional studies should be done - e.g. putative regulatory sequence motifs associated with the neighbors of these highly connected metabolites [39]. Furthermore, most connected metabolites with filtered cofactors can be found in Additional file 3.

\section{Simulations of the three metabolic modes}

$i$ Syn669, together with appropriate physiological constraints, was used as a stoichiometric simulation model by using FBA algorithm [40]. The FBA model simulates steady state behavior by enforcing mass balances constraints for the all metabolic intermediates (Methods). Biomass synthesis, a theoretical abstraction for cellular growth, is considered as a drain of some of these intermediates, i.e. building blocks, into a general biomass component. Different studies have reported that the simulation results do not usually vary drastically when using a common biomass equation for different growth condition $[15,24]$. Nevertheless, experimental efforts should be directed at the depiction of the best precursors and composition that could characterize, at least, the three main growth modes, viz., autotrophy, heterotrophy and mixotrophy, in the scope of recent results [41]. Due to the lack of such data, the present work uses one single biomass equation in the simulations of all three metabolic states (Table 3). Presence of photosynthesis allows $i$ Syn669 to "grow" under the all three metabolic states (i.e., FBA with biomass formation as an objective function results in a feasible solution): carbon dioxide and light (autotrophic), sugars (heterotrophic), carbon dioxide, light and sugars (mixotrophic).

Growth under pure heterotrophy, or dark heterotrophy (in the absence of light) is a subject under study $[42,43]$, being the regular experimental design to give a short light pulse prior to the pure heterotrophic phase (light-activated heterotrophy). Nevertheless, the theoretical flux distribution under heterotrophic conditions is interesting by itself - especially in comparison with the flux distribution in a light-fed energy metabolism. Moreover, fluxes in the heterotrophy mode may help in obtaining insight into the variations under the mixotrophic condition, which is of high relevance for industrial applications [9].

All FBA simulations were carried out under the appropriate constraints so as to match an autotrophic specific growth rate of $0.09 \mathrm{~h}^{-1}$. This growth rate corresponds to a light input of $0.8 \mathrm{mE} \mathrm{g}_{\mathrm{DW}}{ }^{-1} \mathrm{~h}^{-1}$ and to a net
Table 3 iSyn669 Biomass composition

\begin{tabular}{lrll}
\hline Metabolite & $\begin{array}{c}\text { mmole/g } \\
\text { DCW }\end{array}$ & Metabolite & $\begin{array}{c}\text { mmole/g } \\
\text { DCW }\end{array}$ \\
\hline $\begin{array}{l}\text { Amino acids } \\
\text { [38] }\end{array}$ & & $\begin{array}{l}\text { Deoxyribonucleotides } \\
\text { [58] }\end{array}$ & \\
\hline Alanine & 0.499149 & dATP & 0.0241506 \\
\hline Arginine & 0.28742 & dTTP & 0.0241506 \\
\hline Aspartate & 0.234232 & dGTP & 0.02172983 \\
\hline Asparagine & 0.234232 & dCTP & 0.02172983 \\
\hline Cysteine & 0.088988 & Ribonucleotides [1] & \\
\hline Glutamine & 0.255712 & AMP & 0.14038929 \\
\hline Glutamate & 0.255712 & UMP & 0.14038929 \\
\hline Glycine & 0.595297 & GMP & 0.12374585 \\
\hline Histidine & 0.092056 & CMP & 0.12374585 \\
\hline Isoleucine & 0.282306 & Lipids [59] & \\
\hline Leucine & 0.437778 & 16C-lipid & 0.20683718 \\
\hline Lysine & 0.333448 & (9Z)16C-lipid & 0.01573412 \\
\hline Methionine & 0.149336 & 18C-lipid & 0.00351776 \\
\hline Phenylalanine & 0.180021 & (9Z)18C-lipid & 0.03188596 \\
\hline Proline & 0.214798 & (9Z,12Z)18C-lipid & 0.03568367 \\
\hline Serine & 0.209684 & (9Z,12Z,15Z)18C-lipid & 0.01797109 \\
\hline Threonine & 0.246506 & (6Z,9Z,12Z)18C-lipid & 0.05031906 \\
\hline Tryptophan & 0.055234 & (6Z,9Z,12Z,15Z)18C-lipid & 0.01448179 \\
\hline Tyrosine & 0.133993 & Antenna chromophores & \\
\hline Valine & 0.411184 & Chlorophyll a & 0.00820225 \\
\hline Carbohydrates $[61]$ & Carotenoids & \\
\hline Glycosen & 0.01450617 & & \\
\hline
\end{tabular}

Glycogen $\quad 0.01450617$

Biomass composition description with references where the information was retrieved from. All this building blocks with their respective stoichiometric coefficient is converted into one gram of dry cell weight. Biomass equation is reaction Biomass in Additional files 2 and 4.

carbon flux of $3.4 \mathrm{mmol}_{\mathrm{DW}}{ }^{-1} \mathrm{~h}^{-1}$ into the cell, with $\mathrm{HCO}_{3}{ }^{-}$and $\mathrm{CO}_{2}$ as carbon sources. For the sake of comparison across the different conditions, uptake rates for the corresponding carbon sources were matched based on normalization per number of carbon atoms (this does not affect mono-carbon compounds like carbon dioxide and carbonic acid, but has importance in glucose feeding). Results of the subsequent FBA simulations for the three different growth conditions are presented in the following. Some of the reactions that are physiologically relevant for each of the conditions are summarized in Table 4 and Figure 1. Flux values for the rest of the reactions, including the upper and lower bounds are provided in Additional file 4.

\section{Heterotrophy}

Heterotrophy was simulated by considering glucose as the sole carbon source with uptake rate of $0.567 \mathrm{mmol}$ $\mathrm{g}_{\mathrm{DW}}{ }^{-1} \mathrm{~h}^{-1}$, entering the system through $g l c P$ glucose transporter (reaction TRANS-RXN59G-152 in iSyn669). With the purpose of having a pure heterotrophic state, 
Table 4 Comparison of selected fluxes across different growth conditions

\begin{tabular}{|c|c|c|c|c|c|c|c|c|c|c|c|c|c|}
\hline $\begin{array}{l}\text { Reaction } \\
\text { name }\end{array}$ & Autotrophy & $\underset{\text { flux }}{\text { Minimum }}$ & $\underset{\text { flux }}{\text { Maximum }}$ & Mixotrophy & $\underset{\text { flux }}{\text { Minimum }}$ & $\begin{array}{l}\text { Maximum } \\
\text { flux }\end{array}$ & $\begin{array}{c}\text { Dark } \\
\text { Heterotrophy }\end{array}$ & $\underset{\text { flux }}{\text { Minimum }}$ & $\begin{array}{l}\text { Maximum } \\
\text { flux }\end{array}$ & $\begin{array}{c}\text { Light } \\
\text { Heterotrophy }\end{array}$ & $\underset{\text { flux }}{\text { Minimum }}$ & $\underset{\text { flux }}{\text { Maximum }}$ & Reaction description \\
\hline $2.7 .1 .2 a$ & 0 & 0 & 0 & 0.567 & 0.566 & 0.567 & 0.567 & 0.566 & 0.567 & 0.567 & 0.566 & 0.567 & $\begin{array}{c}\text { beta-D-glucose + ATP } \rightarrow \\
\text { beta-D-glucose-6- } \\
\text { phosphate + ADP }\end{array}$ \\
\hline 4.2 .1 .2 & 12.67 & 12.667 & $+\infty$ & 14.67 & 14.657 & $+\infty$ & 0.905 & 0.884 & $+\infty$ & 2.148 & 1.836 & $+\infty$ & malate $\leftrightarrow$ fumarate $+\mathrm{H}_{2} \mathrm{O}$ \\
\hline 5.3.1.6 & 1.201 & 1.2 & $+\infty$ & 1.269 & 1.269 & $+\infty$ & -0.054 & -0.051 & -0.055 & 0.066 & 0.067 & $+\infty$ & $\begin{array}{l}\text { D-ribose-5-phosphate } \leftrightarrow \\
\text { D-ribulose-5-phosphate }\end{array}$ \\
\hline UUQ & 0.8 & 0 & 0.8 & 0.8 & 0 & 0.8 & 0 & 0 & 0 & 0.8 & 0 & 0.8 & $\begin{array}{c}\mathrm{PSII}^{*}+\mathrm{UQ}+2 \mathrm{H}+\rightarrow \mathrm{PSII} \\
+\mathrm{UQH} 2\end{array}$ \\
\hline-1.6 .5 .3 & 0 & 0 & $+\infty$ & 0 & 0 & $+\infty$ & 2.134 & 0 & $+\infty$ & 0 & 0 & $+\infty$ & $\begin{array}{c}\mathrm{NADH}+\mathrm{UQ}+7 \mathrm{H}+\rightarrow \\
\mathrm{NAD}++\mathrm{UQH} 2+4 \mathrm{H} \\
+ \text { +peribac }\end{array}$ \\
\hline-3.6 .3 .14 & 38.348 & 15.7 & $+\infty$ & 21.727 & 21.7 & $+\infty$ & 4.98 & 4.95 & $+\infty$ & 6.292 & 6.281 & $+\infty$ & $\begin{array}{c}3 \mathrm{H}+\text { _peribac }+ \text { phosphate } \\
\mathrm{O} 4 \mathrm{P}+\mathrm{ADP} \leftrightarrow 3 \mathrm{H}++ \\
\mathrm{H} 2 \mathrm{O}+\mathrm{ATP}\end{array}$ \\
\hline 6.2 .1 .1 & 0.008 & $-\infty$ & $+\infty$ & -30.017 & $-\infty$ & $+\infty$ & -2.124 & $-\infty$ & $+\infty$ & -4.635 & $-\infty$ & $+\infty$ & $\begin{array}{c}\text { coenzyme A + acetate + } \\
\text { ATP } \leftrightarrow \text { acetyl-CoA }+ \\
\text { diphosphate + AMP }\end{array}$ \\
\hline
\end{tabular}

Units in $\mathrm{mmol} \mathrm{gDw}^{-1} \mathrm{~h}^{-1}$. 2.7.1.2a, glucokinase, is the reaction that phosphorylates beta-D-glucose upon entrance in the cell, marking the start of the glycolysis. The flux direction changes can be seen in reaction 4.2.1.2, fumarate hydratase, from TCA cycle and 5.3.1.6, ribose-5-phosphate isomerase, from the pentose phosphate pathway._UQ and _ 1.6.5.3 are reactions that reduce UQH2 from photosystem II or NADH oxidation, respectively, causing a pumping of protons to the thylakoid. _3.6.3.14 is the ATP synthase that forms ATP shuttling protons from the thylakoid to the cytosol. 6.2.1.1, acetate-CoA ligase, is the reaction that generates acetyl-CoA from acetate and coenzyme A, that would be a major flux hub in an ethanol-producing strain, standing as the first step of fermentation. 


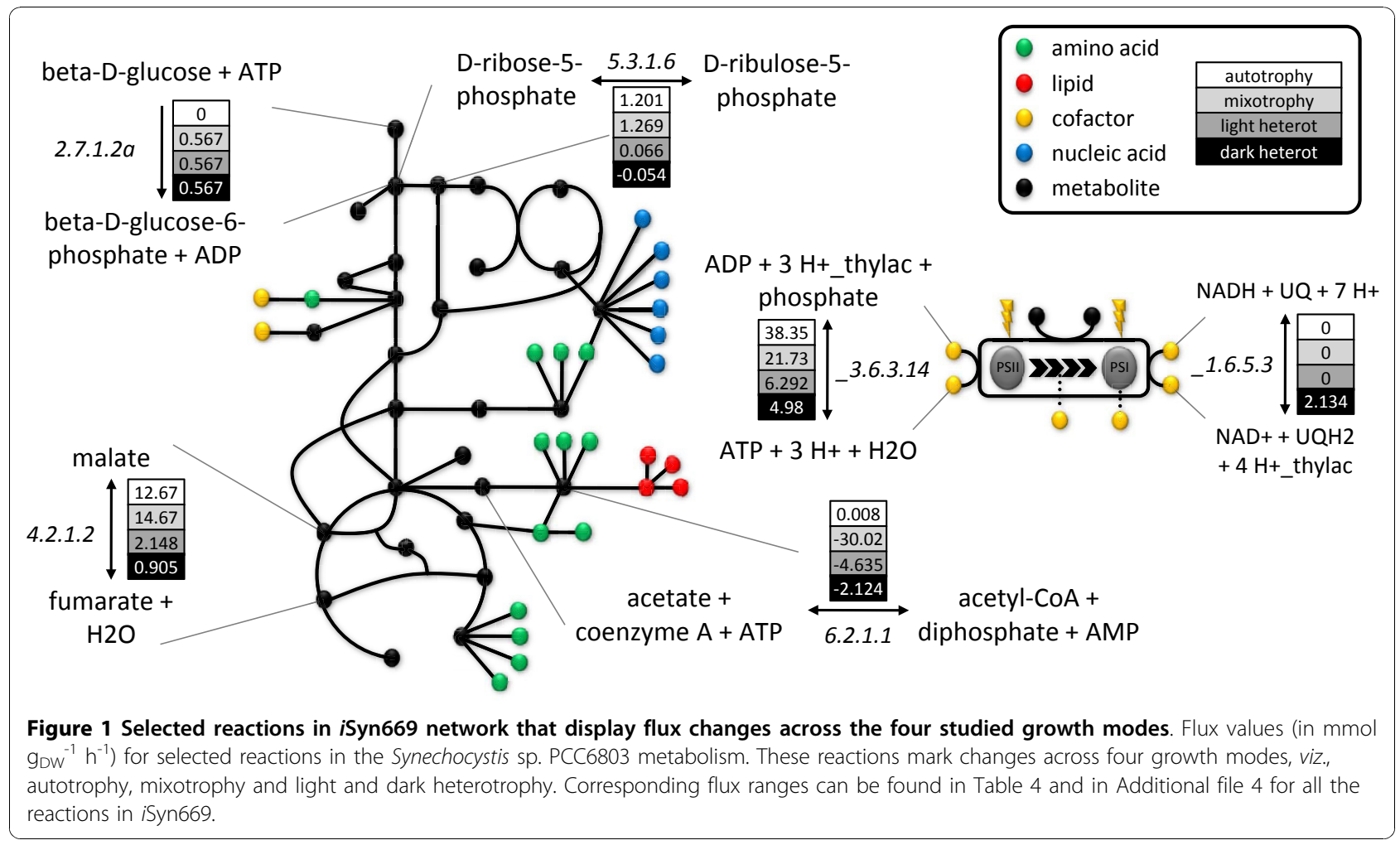

photon uptake rate was constrained to 0 ; this caused photosynthesis fluxes to be shut down. In this case, glucose will be the source for the formation of carbon backbones for the building blocks of the cell, depicted in the biomass equation. The glycolytic and the oxidative mode of the pentose phosphate pathway were found to be active. Oxidative pentose phosphate pathway is the major pathway for glucose catabolism as was reported in reference [44]. PEP carboxylase (reaction 4.1.1.31 in iSyn669) is the main anaplerotic flux to the TCA cycle. Carbon fixation efficiency is around 60\%, the rest being released in the form of $\mathrm{CO}_{2}$, as reported in our previous work [9].

In contrast to dark heterotrophy, if a light-activated heterotrophy simulation is run, light enters the system and $\mathrm{RuBisCO}$ enzyme is active (reaction 4.1.1.39), fixing all the $\mathrm{CO}_{2}$ that was released in dark heterotrophy, boosting carbon efficiency to a theoretical $100 \%$. In this case, global flux distribution as well as flux ranges resemble that of autotrophy more than that of the dark heterotrophy. Carbon skeletons are still produced through glycolysis and $\mathrm{NAD}(\mathrm{P}) \mathrm{H}$ is reduced along the glycolysis, pyruvate metabolism and TCA cycle. On the other hand, pentose phosphate pathway has shifted to the reductive mode due to $\mathrm{RuBisCO}$ activation and the corresponding flux is increased in magnitude. Carbon fixation happens at the $\mathrm{RuBisCO}$ level, thereby assimilating the $\mathrm{CO}_{2}$ produced by the glucose metabolism, and the production of ATP and NADPH through photosynthesis relieves the oxidative phosphorylation from draining NADPH to generate ATP.

\section{Autotrophy}

Photoautotrophy was initially simulated considering an illumination of $0.15 \mathrm{mE} \mathrm{m}^{-2} \mathrm{~s}^{-1}$. Assuming that the mass of a typical Synechocystis sp. PCC6803 cell is 0.5 pg [45] and its radius is $1.75 \mu \mathrm{m}$ [46], we estimated that the theoretical maximum illumination is $41563.26 \mathrm{mE} \mathrm{g}_{\mathrm{DW}^{-1}}$ $\mathrm{h}^{-1}$. An additional optimization step was performed in order to estimate physiologically meaningful photon uptake values that are closer to the experimental measurements [24]. First, carbon uptake rate was found that resulted in a specific growth rate of $0.09 \mathrm{~h}^{-1}$, while the light intake was unconstrained. Next, the growth rate was constrained to this value and the second optimization problem was solved where light uptake was minimized. This minimization resulted in photon uptake for photosystem I (reaction_lightI) and photosystem II (reaction_lightII) being $0.8 \mathrm{mE} \mathrm{g}_{\mathrm{DW}}{ }^{-1} \mathrm{~h}^{-1}$. Carbon sources used in simulating photoautotrophy conditions were carbon dioxide and carbonic acid, and its entrance to the system was mediated by $\mathrm{RuBisCO}$ (reaction 4.1.1.39 in $i$ Syn669) and carbonic anhydrase (reaction 4.2.1.1b) respectively. As $i$ Syn 669 biomass equation encompasses all essential metabolite precursors, these will be the sinks of our network, while photons, carbon dioxide and/or carbonic acid will be the sources. Thus 
autotrophic fluxes will flow in the gluconeogenic direction and through the Calvin cycle, which is the reductive mode of the pentose phosphate pathway. PEP carboxylase is the main anaplerotic flux to the TCA cycle and glyoxylate shunt is inactive.

\section{Mixotrophy}

Photons, carbon dioxide and glucose are independent feed fluxes in this simulation. These fluxes entered the system through the same reactions as described for the previous growth modes. Carbon source presents, in this case, one more degree of freedom than in the rest of the conditions. In order to keep a comparative criterion across conditions, we normalized $\mathrm{CO}_{2}$ and glucose inputs to the same carbon uptake flux as in the case of the autotrophy and the heterotrophy. Photon uptake rates were also normalized in a similar manner to match the autotrophic state. Having the same metabolic sinks as the two previous modes and the sources from the both of them, it is logical to think that the resulting flux distribution will be a mixture of the autotrophic and heterotrophic simulations. Indeed, we observed that the mixotrophic flux distribution lies inbetween the previous two states, being a bit closer to the heterotrophy. Glycolysis is present and glyoxylate is shut down; an active photosynthesis is present, oxidative phosphorylation is less stressed than in heterotrophy as the energy can be produced from the photon uptake; and Calvin cycle is active, as carbon sources are $\mathrm{CO}_{2}$ and glucose.

\section{Flux variability analysis}

Flux balance analysis presented above guarantees to find the optimal objective function value (biomass formation rate). However, the predicted intra-cellular flux distribution is not necessarily unique due to the

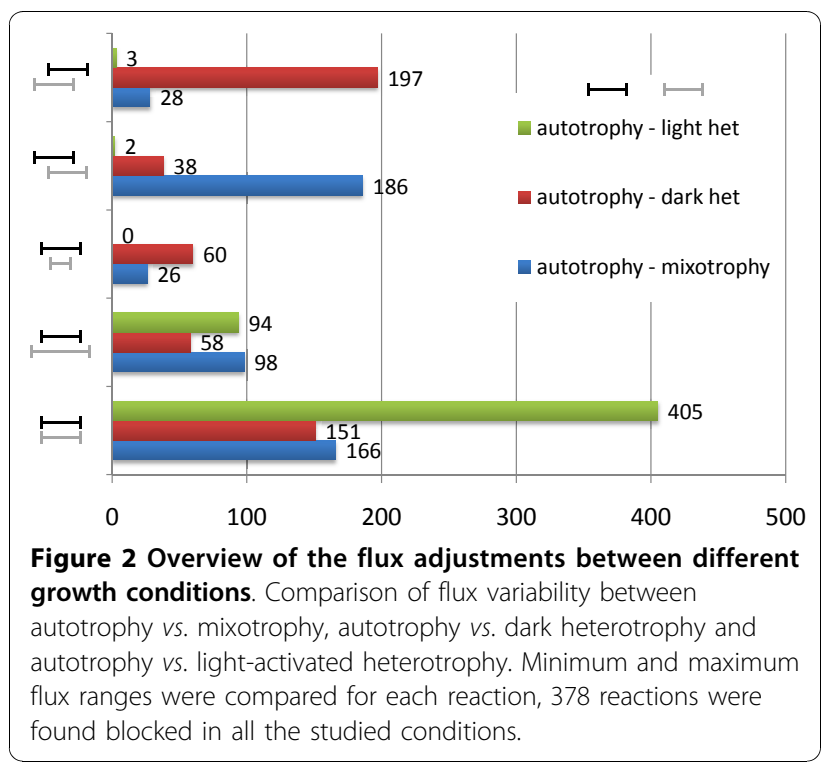

presence of multiple pathways that are equivalent in terms of their overall stoichiometry. Thus, often the system exhibits multiple optimal solutions and further elucidation requires additional constraints based on experimental evidences (e.g. carbon labeling data). Alternatively, physiological insight can be still obtained by studying the variability at each flux node given the objective function value - a procedure referred to as flux variability analysis. In order to gain insight into the flux changes underlying the changes in the Synechocystis metabolism due to (un)availability of light, we have compared the autotrophic growth with the other two by using flux variability analysis (Figure 2). Interestingly, autotrophy permits an overall broader flux landscape than heterotrophy (let it be dark or light-activated). On the other hand autotrophic flux ranges are in general narrower than the mixotrophic ranges. Figure 1 and Table 4 depict some of the physiologically relevant reactions for which the feasible flux range differs across conditions. These include glucokinase from glycolysis, fumarate hydratase from TCA cycle, ribose-5-phosphate isomerase from pentose phosphate pathway, NADH dehydrogenase from oxidative phosphorylation or photosystem II oxidation. These reactions mark the key nodes in the metabolism network that must be appropriately regulated in order to adapt in response to the available energy/carbon source. Mechanisms underlying such changes will be of particular interest not only for biotechnological applications but also from the biological point of view. As a glimpse of the detailed flux (re-) distributions in each of the studied growth conditions, Additional file 5 describes fluxes in the pyruvate metabolism.

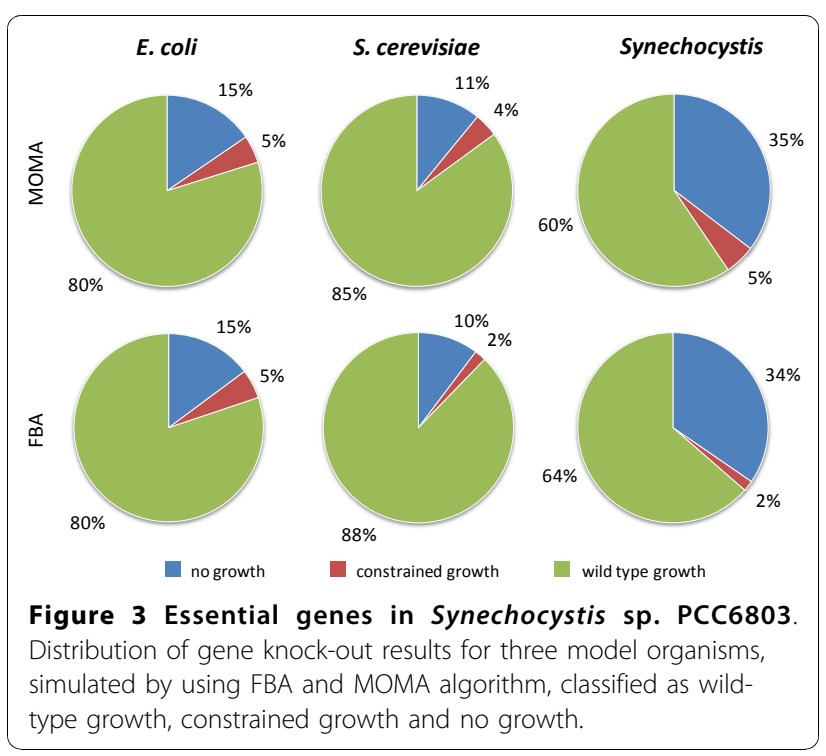




\section{Gene/Reaction knock-out analysis}

The comprehensive set of reconstructed biochemical equations of $i$ Syn 669 and FBA simulations enabled us to further analyze the characteristics and potential of the Synechocystis metabolic network. This can be oriented towards the study of the reactions (and thereby the corresponding genes) that are necessary for the growth, or to in silico metabolic engineering for identification of targets for maximization of a given metabolite of socioeconomic interest.

\section{Essential Genes}

iSyn669 network consists of 790 metabolites and 882 reactions. Among these, 350 genes (36\% of the total, Figure 3) were found to be necessary for the formation of the biomass under the mixotrophic growth conditions by using FBA and MOMA algorithms. This set of genes can be divided in to two categories: i) essential genes, deletion of which completely inhibits biomass growth (304 genes, $34 \%$ of the total, with FBA): and ii) genes deletion of which causes a reduced growth rate (46 genes, $2 \%$ of the total, with FBA). The set of 304 essential genes can be understood as the core of the metabolism, as deleting them would produce an unviable organism. The results based on MOMA algorithm essentially tally these numbers: 311 essential genes, $35 \%$ of the total, and 45 that cause a reduced growth rate, $5 \%$ of the total, (Additional file 6).

Interestingly, if we compare the proportion of the essential genes under FBA simulation in the metabolic networks of $E$. coli (187 genes, $15 \%$ of the total) [38] and Saccharomyces cerevisiae (148, 10\% of the total) [32] with iSyn669, we find that Synechocystis has a significantly larger fraction of metabolic genes whose deletion obliterates biomass formation (304 genes, 34\% of the total). One possible explanation for the difference in the relative proportion of essential genes in these three organisms would be an incomplete/incorrect annotation of the genome of Synechocystis sp. PCC6803. For example, if only one of the isoenzymes corresponding to a reaction is annotated, the corresponding in silico knockout will result in a false negative prediction. It is important to note that the computational predictions of gene essentiality based on FBA are highly dependent on the growth medium used for the simulations. Thus, the comparison across different species may not be straightforward. Moreover, it is also possible that the natural growth conditions of Synechocystis may have dictated selection for a relatively high proportion of essential genes. Such hypotheses need careful consideration of several factors and are beyond the scope of this work.

\section{Production of value-added compounds}

Synechocystis sp. PCC6803 is considered as a candidate photobiological production platform - it can potentially produce molecules of interest by using $\mathrm{CO}_{2}$ and light
[6]. To this end, iSyn669 can be used to perform simulations, not only for assessing the feasibility of producing a given compound, but also to identify potential metabolic engineering targets towards improved productivity. For example, FBA simulations can help in estimating maximum theoretical yields for the products/intermediates of interest. A product of obvious interest is hydrogen. In our previous work [9], we have estimated maximum theoretical hydrogen production values that are far from the current state of experimental reports. In silico studies can direct the efforts and counsel the scientists towards a hydrogen producing cyanobacteria that could be of impact. iSyn669 predicts, in autotrophic conditions, a theoretical $\mathrm{H}_{2}$ evolution rate of $0.17 \mathrm{mmol}$ $\mathrm{g}_{\mathrm{DW}}{ }^{-1} \mathrm{~h}^{-1}$ obliterating biomass growth. Else, the stoichiometry permits the evolution of $0.156 \mathrm{mmol} \mathrm{g}_{\mathrm{DW}}{ }^{-1} \mathrm{~h}^{-}$ ${ }^{1}$ of hydrogen with a biomass growth of $10 \%$ of the wild type $\left(0.007 \mathrm{mmol} \mathrm{g}_{\mathrm{DW}}{ }^{-1} \mathrm{~h}^{-1}\right)$.

Succinate is an important metabolite for its biotechnological applications as well as for being a metabolite that bridges the TCA cycle with the electron transfer chain. As an example of the usefulness of the present metabolic model we have designed an in silico metabolic engineering strategy to improve the production of succinate. The underlying idea is to design a succinate overproducing metabolic network (through reaction knockout simulations), whereas the intracellular fluxes are distributed so as to maximize the biological objective function (e.g. growth) [47]. To this end, OptGene algorithm [37] was used together with Minimization Of Metabolic Adjustment (MOMA) [17] as a biological objective function. MOMA has been reported to provide better description of flux distributions in mutants or under un-natural growth conditions as opposed to FBA. A design objective function which copes with the metabolite of interest, succinate, has been determined maintaining the biological objective function as the biomass formation.

OptGene simulations for single, double and triple knock-out strategies were performed to obtain solutions with improved succinate production, but without drastically diminishing the biomass production. We used mixotrophic conditions, for which wild type optimal growth rate was $0.17909 \mathrm{mmol} \mathrm{g}_{\mathrm{Dw}}{ }^{-1} \mathrm{~h}^{-1}$. The best single knock-out was found to be the mutant of pyruvate kinase (reaction 2.7.1.40c in iSyn669 and genes sll0587 and sll1275) that has a succinate evolution of 0.5695 $\mathrm{mmol} \mathrm{g}_{\mathrm{DW}}{ }^{-1} \mathrm{~h}^{-1}$ with a growth rate of $0.0714 \mathrm{mmol}$ $g_{D W}{ }^{-1} h^{-1}$. Blocking this reaction, preventing pyruvate and phosphoenolpyruvate from using GTP and GDP would drive a high increase in succinate production. The flux between pyruvate and phosphoenolpyruvate can still be accomplished with reactions 2.7.1.40a and 2.7.9.2, but using ATP and ADP as cofactors. Double 
deletion did not improve the results from the single knock-out strain, evolving the same succinate production with the same growth rate. The best triple knockout was found to be the combination of pyruvate kinase (reaction 2.7.1.40c in iSyn669 and genes slloo18 and sll0587), fructose-bisphosphate aldolase (reaction 4.1.2.13b in iSyn669 and genes slr0943 and sll1275) and succinate dehydrogenase (reaction _1.3.99.1 in iSyn669 and genes sllo823, sll1625 and slr1233). This simulated strain has a succinate evolution of $0.6999 \mathrm{mmol} \mathrm{g}_{\mathrm{DW}}{ }^{-1}$ $\mathrm{h}^{-1}$ with a growth rate of $0.0688 \mathrm{mmol} \mathrm{g}_{\mathrm{DW}}{ }^{-1} \mathrm{~h}^{-1}$. This design combines the blocking of the oxidation of succinate on the electron chain transfer through succinate dehydrogenase with the prevention of using GTP between pyruvate and phosphoenolpyruvate and the lack of an aldolase needed in the reductive mode of the pentose phosphate pathway. This leads to a situation where flux is directed to TCA cycle in order to meet with an overproduction of succinate.

These studies on knock-outs are reaction centered, even though the in vivo knock-out building will ultimately be through gene manipulations. This is the reason underlying the fact that we found 2.7.1.40c knockout as the best result. This design would hint at the idea of selection of a mutated pyruvate kinase protein specific for ATP cofactor. This may be difficult to achieve on the bench, but has high biotechnological expectations.

\section{iSyn669 as a data integration scaffold}

Apart from the flux simulations, another important problem in the field of metabolic systems biology that can be addressed by using reconstructed genome-scale models is the integration of the different genome-wide biomolecular abundance datasets, i.e. omics datasets, such as transcriptome and metabolome. An example of algorithms for carrying out such an integrative analysis through the use of genome-scale metabolic networks is Reporter Features [48,49]. Reporter algorithm allows integration of omics data with bio-molecular interaction networks, thereby allowing identification of cellular regulatory focal points (i.e. reporter features), for instance reporter metabolites as regulatory hubs in the metabolic network.

In this work, Reporter Features software was used to integrate transcriptional information over the reconstructed Synechocystis sp. PCC6803 network allowing us to infer regulatory principles underlying metabolic flux changes following shifts in growth mode. In particular, we analyzed the data from a work [50] that reports the transcriptional changes caused in Synechocystis sp. PCC6803 by shifts from darkness to illumination conditions and back. As it can be understood from the rationale beneath the metabolic capabilities of this cyanobacterium, the presence or absence of light drives big changes in the flux distribution through the network, as discussed in the previous sections. We have focused our study on the relationship between the transcription of Synechocystis sp. PCC6803 genes and the reactions of the metabolic network. Associations between genes and reactions were identified, listing all the genes that performed or were involved in a specific reaction. With this information and the metabolic model, Reporter Features analysis was carried out. In brief, the analysis helped to identify metabolites around which the transcriptional changes are significantly concentrated. These metabolites are termed reporter metabolites as they represent key regulatory nodes in the network.

Gill et al [50] designed the experiment so that Synechocystis was grown to mid-exponential phase $\left(\mathrm{A}_{730}=\right.$ 0.6 to 0.8 ). Then, the lights were extinguished and RNA samples were taken after $24 \mathrm{~h}$ in the dark (full dark). Illumination was then turned back on for 100 min (transient light), followed immediately by an additional 100 min in the dark (transient dark).

We were interested in two aspects of this study: i) to identify metabolites around which regulation is centered during the light regime transitions; and ii) to find the metabolic genes that were collectively significantly coregulated across these transitions [49].The analysis was divided in three parts: an analysis of the data arrays from the whole experimental profile ("all time points"), an analysis of the shift from darkness to a light environment ("dark to light") and from light back to dark ("light to dark"). For a study of the overall genome and its light regulation, refer to Gill et al [50]. In this study, as the relationship between the metabolism and this regulation was investigated, genes with no direct relationship to a metabolic reaction were not considered. Distributions of the genes across KEGG Orthologies related to the metabolism altered with the light shift are depicted in Table 5.

\section{All time points}

When all seven arrays were used, reporter metabolites were found to be quite scattered across the metabolism spanning several metabolic pathways, and thus offering a global view of the transcriptional response in the metabolic network (see Figure 4a and Table 6a). Presence of some amino acids (L-tyrosine, L-isoleucine), nucleic acids and its precursors (GTP, dihydroorotate), carbon metabolism metabolites (D-ribulose-5-phosphate, succinyl-CoA), lipids precursors (myo-inositol, D-myoinositol 3-monophosphate), cofactors (thioredoxin, p-aminobenzoate) and photosynthesis metabolites (plastocyanin) pictures a scenario of a global regulation throughout the different metabolic pathways.

By using the metabolic sub-network search algorithm, we found 212 genes that have their expression changed 
Table 5 KEGG orthology groups for the metabolic genes altered with the light shift.

\begin{tabular}{|c|c|c|c|c|c|c|}
\hline & \multicolumn{2}{|c|}{ All time points } & \multicolumn{2}{|c|}{ Dark to Light } & \multicolumn{2}{|c|}{ Light to Dark } \\
\hline & $\begin{array}{l}\text { Number } \\
\text { of genes }\end{array}$ & $\%$ & $\begin{array}{l}\text { Number } \\
\text { of genes }\end{array}$ & $\%$ & $\begin{array}{l}\text { Number } \\
\text { of genes }\end{array}$ & $\%$ \\
\hline Energy Metabolism & 128 & 60.38 & 128 & 51.82 & 127 & 61.65 \\
\hline Amino Acid Metabolism & 25 & 11.79 & 31 & 12.55 & 24 & 11.65 \\
\hline Carbohydrate Metabolism & 24 & 11.32 & 28 & 11.33 & 23 & 11.16 \\
\hline Metabolism of Cofactors and Vitamins & 13 & 6.13 & 26 & 10.53 & 12 & 5.83 \\
\hline Nucleotide Metabolism & 12 & 5.66 & 23 & 9.32 & 12 & 5.83 \\
\hline Lipid Metabolism & 7 & 3.3 & 5 & 2.02 & 6 & 2.91 \\
\hline Membrane Transport & 3 & 1.42 & 4 & 1.63 & 2 & 0.97 \\
\hline $\begin{array}{c}\text { Biosynthesis of Secondary } \\
\text { Metabolites }\end{array}$ & 0 & 0 & 1 & 0.4 & 0 & 0 \\
\hline $\begin{array}{l}\text { Biosynthesis of Polyketides } \\
\text { and Nonribosomal Peptides }\end{array}$ & 0 & 0 & 1 & 0.4 & 0 & 0 \\
\hline Total & 212 & 100 & 247 & 100 & 206 & 100 \\
\hline
\end{tabular}

across the arrays and that have a relationship with the metabolites of $i$ Syn 669 network. Furthermore, 50 genes were identified that are strongly co-regulated all along the profile of the experiment (Additional File 7, section a). This set of genes is characterized in two groups. The first set consists of the genes from photosynthesis (93.85\%) and oxidative phosphorylation (6.15\%). The second set is representative of a variety of genes from different pathways such as amino acid metabolism

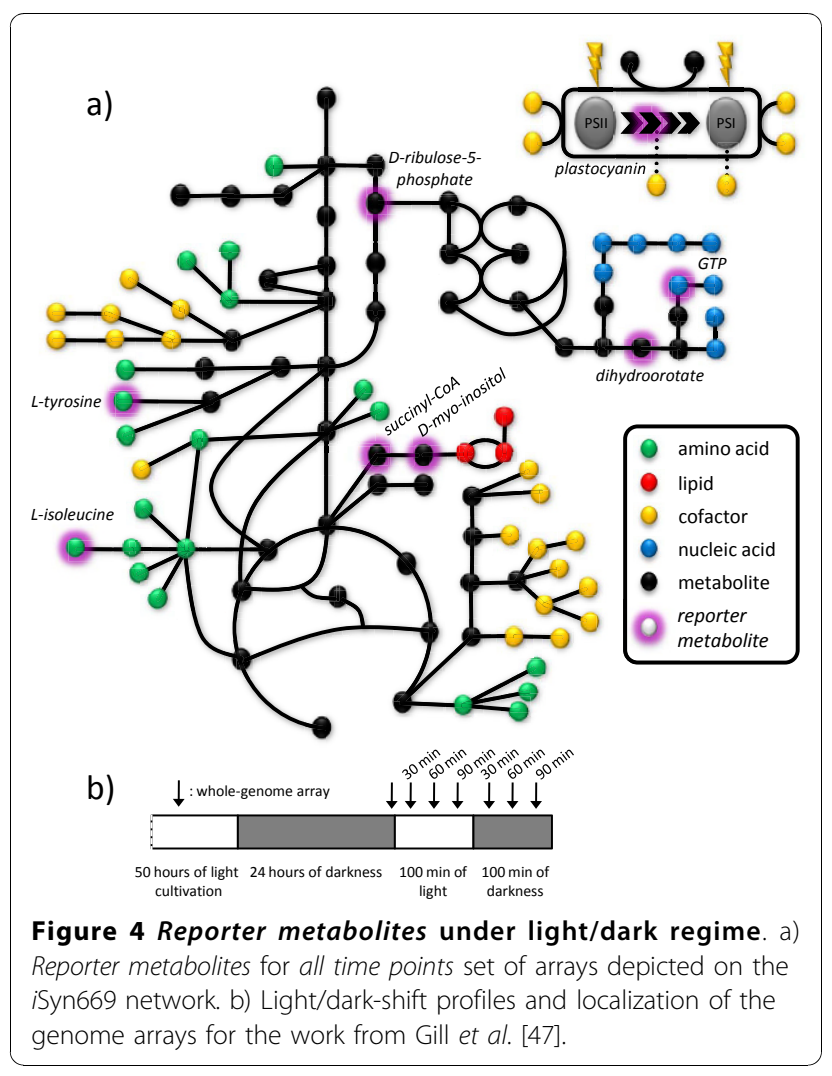

(39\%), carbohydrate metabolism (22\%), nucleotide metabolism (13\%), nitrogen metabolism (13\%) and metabolism of cofactors $(9 \%)$ that globally regulates the entire metabolic network (see Table 5 for further details).

It can be expected that an experimental design like the one we have based our work on, which combines a shift from dark to light with a shift back to darkness, will encompass an important part of the regulatory changes the cell is undergoing in its natural habitat. In a glucose-deficient environment, the presence or absence of light is the main condition around which the Synechocystis metabolism gravitates [9]. Indeed, one of the co-regulated sets consists of the genes coding for the proteins that work on, and around, the thylakoid membrane, let it be photosynthesis or oxidative phosphorylation genes.

\section{Dark to light}

Next, we considered the arrays that represent the shift from darkness to light, the first three arrays (from " 24 hours of darkness" array to "60 minutes of light" array). Reporter metabolites were found to be largely within the nucleotide and amino acid metabolism (Table 6b). Some cofactors were also identified as regulation hubs like tetrahydrofolate, thioredoxin and adenosylcobinamide.

Sub-network search yielded set of 247 genes that have their expression changed across the first three arrays and that are related with $i$ Syn 669 reactions. Furthermore, 84 genes were identified that are strongly coregulated across the three arrays (Additional File 7, section b). This set of genes cover photosynthesis (25\%), oxidative phosphorylation (24\%), amino acid metabolism (11\%), carbohydrate metabolism (11\%), nucleotide metabolism (10\%) and metabolism of cofactors (10\%).

This set of data arrays are indeed a good example of a cell's metabolic machinery starting up. After a 24 hour period in darkness where cell density did not 
Table 6 Reporter metabolites for the light shift experiment.

\begin{tabular}{|c|c|c|c|c|c|}
\hline a) & & b) & & c) & \\
\hline Metabolite & $\begin{array}{l}\text { Number of } \\
\text { neighbors }\end{array}$ & Metabolite & $\begin{array}{l}\text { Number of } \\
\text { neighbors }\end{array}$ & Metabolite & $\begin{array}{c}\text { Number of } \\
\text { neighbors }\end{array}$ \\
\hline All time points & & Dark to Light & & Light to Dark & \\
\hline L-tyrosine & 4 & N-carbamoyl-L-aspartate & 3 & 5-phosphoribosyl-N-formylglycineamidine & 3 \\
\hline $\begin{array}{l}\mathrm{N} \text {-carbamoyl-L- } \\
\text { aspartate }\end{array}$ & 3 & dihydroorotate & 3 & diphosphate & 76 \\
\hline dTDP & 4 & $\begin{array}{l}\text { 5-phosphoribosyl 1- } \\
\text { pirophosphate }\end{array}$ & 9 & a 1,4-alpha-D-glucan_n & 2 \\
\hline L-isoleucine & 3 & L-valine & 3 & a 1,4-alpha-D-glucan_n1 & 2 \\
\hline $\begin{array}{l}\text { D-ribulose-5- } \\
\text { phosphate }\end{array}$ & 4 & $\begin{array}{l}\text { 5-phospho-ribosyl- } \\
\text { glycineamide }\end{array}$ & 3 & $\begin{array}{l}\text { UDP-N-acetylmuramoyl-L-alanyl-D-glutamyl-meso-2,6- } \\
\text { diaminoheptanedioate }\end{array}$ & 2 \\
\hline $\begin{array}{l}\text { D-myo-inositol (3)- } \\
\text { monophosphate }\end{array}$ & 2 & $\begin{array}{l}\text { O-phospho-L- } \\
\text { homoserine }\end{array}$ & 2 & pyridoxine-5'-phosphate & 2 \\
\hline myo-inositol & 2 & $\begin{array}{l}\text { peptidylproline (omega } \\
=180 \text { ) }\end{array}$ & 4 & (E, E)-farnesyl diphosphate & 3 \\
\hline L-valine & 3 & $\begin{array}{l}\text { peptidylproline (omega } \\
=0 \text { ) }\end{array}$ & 4 & GMP & 6 \\
\hline succinyl-CoA & 3 & $\begin{array}{l}\text { indole-3-glycerol- } \\
\text { phosphate }\end{array}$ & 2 & phosphoribosylformiminoAICAR-phosphate & 2 \\
\hline adenosine & 2 & $\begin{array}{l}\text { 5-aminoimidazole } \\
\text { ribonucleotide }\end{array}$ & 3 & L-aspartyl-4-phosphate & 2 \\
\hline GTP & 13 & $\begin{array}{l}\text { tetrahydrofolate } \\
\text { cofactors }\end{array}$ & 8 & pantothenate & 2 \\
\hline thioredoxin & 11 & GTP & 13 & $\begin{array}{l}\text { undecaprenyl-diphospho-N-acetylmuramoyl-L-alanyl-D- } \\
\text { glutamyl-meso-2,6-diaminopimeloyl-D-alanyl-D-alanine }\end{array}$ & 2 \\
\hline $\begin{array}{l}\text { thioredoxin } \\
\text { disulfide }\end{array}$ & 11 & $\begin{array}{l}\text { L-glutamate gamma- } \\
\text { semialdehyde }\end{array}$ & 2 & $\begin{array}{l}\text { MurAc(oyl-L-Ala-D-gamma-Glu-L-Lys-D-Ala-D-Ala)- } \\
\text { diphospho-undecaprenol }\end{array}$ & 2 \\
\hline p-aminobenzoate & 2 & inosine-5'-phosphate & 5 & $\begin{array}{l}\text { undecaprenyl-diphospho-N-acetylmuramoyl-L-alanyl-D- } \\
\text { glutamyl-L-lysyl-D-alanyl-D-alanine }\end{array}$ & 2 \\
\hline acetylphosphate & 2 & $\begin{array}{l}\text { pantetheine } 4^{\prime}- \\
\text { phosphate }\end{array}$ & 2 & L-aspartate-semialdehyde & 2 \\
\hline glycine & 7 & $\begin{array}{l}\text { UDP-N-acetylmuramoyl- } \\
\text { L-alanyl-D-glutamate }\end{array}$ & 2 & 5-phospho-ribosyl-glycineamide & 3 \\
\hline succinate & 7 & phytoene & 2 & 5'-phosphoribosyl-N-formylglycineamide & 4 \\
\hline dihydroorotate & 3 & thioredoxin & 11 & sulfur & 2 \\
\hline$P C$ & 12 & thioredoxin disulfide & 11 & glycine & 7 \\
\hline
\end{tabular}

Reporter metabolites for each set of arrays analysed with Reporter Features software.

change (see Figure 1 in Gill et al [50]), light enters the system and the cell starts to synthesize new bio-molecules, mostly nucleotides so it can copy its genetic material and amino acids to build up proteins.

\section{Light to dark}

Finally, we considered the arrays that represent the shift from light to dark, data from "90 minutes of light" array to "60 minutes of dark" array. Similar to the previous case study, reporter metabolites were found to be focused on the nucleotide and amino acid metabolism (Table 6c). Additionally, the presence of metabolite $a$ 1,4-alpha-D-glucan_n and its cognate a 1,4-alpha-D-glucan_n1 also stands out as they are involved in carbon reserves catabolism and anabolism.
With the help of the sub-network search, 133 genes were identified as being significantly co-regulated across those three arrays (Additional File 7, section c). This set comprises of the genes from photosynthesis (34\%), oxidative phosphorylation (26\%), amino acid metabolism (12\%), carbohydrate metabolism (12\%), nucleotide metabolism (7.5\%) and metabolism of cofactors (4.5\%).

This last set of data array is a scenario where metabolism is being shut down, as a consequence of the darkness and lack of carbohydrate source. Without light, photosynthesis is blocked and carbon fixation is nearly obliterated. Cells strive to build up carbon reserves (hence the presence of a 1,4-alpha-D-glucan_n as a reporter metabolite) and oxidative phosphorylation is the 
main energy pathway that remains present. Regulation is centered on the energy metabolism shift $(60 \%$ of the total co-regulated sub-network), withholding amino acids and nucleotide precursors and keeping the cofactors available in a low-profile metabolism.

\section{Conclusions}

We have successfully reconstructed a genome-scale metabolic network for Synechocystis sp. PCC6803, called $i$ Syn669, which allows simulating production of all the metabolic precursors of the organism. The metabolic reconstruction represents an up-to-date database that encompasses all knowledge available in public databases, scientific publications and textbooks on the metabolism of this cyanobacterium.

From the annotation publicly available, our metabolic network includes 882 metabolic reactions and 790 metabolites, as well as the information from 669 genes that have some relationship with the metabolic reactions. This model is the most complete and comprehensive work for Synechocystis sp. PCC6803 to date, which has its potential as the photosynthetic model organism. Interestingly, the reconstruction identified 79 reactions that should be present in the metabolism but with no cognate gene discovered yet; this should direct experimental work at the discovery of these genes. Topological characteristics of the network resemble those of other reconstructed microbial metabolic networks and thus provide an additional input for the analysis of their structural and organizational properties from evolutionary perspective.

Applicability of $i$ Syn669 metabolic model was demonstrated by using a variety of computational analyses. Flux balance analysis was applied in order to simulate the three physiologically important growth conditions of cyanobacteria, viz., heterotrophic, mixotrophic and autotrophic. Our metabolic model was capable of simulating the production of the monomers or building blocks that build up the cells, in the range that is in agreement with the reported growth experiments. Our photosynthetic metabolic model includes all of the central metabolic pathways that previous works [23-25] considered. Regarding the parts from our model that overlap with the previous works (part of the central carbon metabolism), the predictions for the flux directionality changes following light shift match between those models and $i$ Syn669. In fact, iSyn669 expands the flux study to all the pathways described in the Synechocystis sp. PCC6803 genome annotation. Further work should be directed at the definition of a detailed and descriptive biomass cell composition, so as to have a better representation of the biomass equation for simulation purposes.
Single reaction/gene knock-out simulations revealed 311 genes that are essential for the survival. Bearing in mind the distance from the efforts taken in the annotation of the genome of the bacteria and yeast models to that of the cyanobacterium, our study shows that Synechocystis sp. PCC6803 has a larger fraction of genes that are essential for producing biomass, as opposed to Escherichia coli and Saccharomyces cerevisiae. Further investigation of the causes for this difference will be of definite interest in understanding the genome annotation and/or the evolution of the metabolic network of Synechocystis.

Evaluation of the theoretical potential of this organism to produce hydrogen was assessed, in support of the efforts directed to this direction from several groups and scientific council initiatives. Present hydrogen production projects are far from the theoretical potential, but efforts in this field can trigger a very significant increase of the present hydrogen evolution rates in Synechocystis sp. PCC6803 or other photobiological production platforms candidates, e.g. Chlamydomonas reinhardtii, Nostoc punctiforme and Synechococcus species.

Suitability of the presented model for performing in silico metabolic engineering analysis was demonstrated by using OptGene software framework. Furthermore, we also show that iSyn669 can be used as a scaffold to integrate network-wide omics data. As a case study, we identified key reporter metabolites around which regulation during light shifts is organized, as well as gene subnetworks that were co-regulated across the light conditions.

Altogether, the genome-scale metabolic network of Synechocystis sp. PCC6803 (iSyn669) will be a valuable tool for the applied and fundamental research of Synechocystis sp. PCC6803, as well as for the broad field of metabolic systems biology. iSyn669 represents an important step for the integration of tools and knowledge from different disciplines towards development of photo-biological cell factories.

\section{Methods}

\section{Metabolic network reconstruction}

Pathway Tools software [33] was used to construct a Synechocystis-specific database of genes, proteins, enzymes and metabolites. Synechocystis sp. PCC6803 genome and annotation files were downloaded from NCBI Entrez Genome repository as of date 10 of September of 2008 [51]. Pathway tools retrieved a first version of the network, which had to be checked with different kinds of databases depending on the information they bear. Databases used towards this purpose included Enzyme nomenclature database [34], KEGG pathway database [27], BioCyc genome database [26], 
BRENDA Enzyme database [28] and UniProt protein database [29].

Parts that characterize Synechocystis network, like the incomplete TCA cycle [52,53], the presence of the glyoxylate shunt [35], the interconnected photosynthesis and oxidative phosphorylation [54] or the cyclic and non-cyclic electron transport related to these latter processes [55-57], were accounted for in detail.

At the end of the reconstruction process, four kinds of relationships were present in the database: reaction with cognate genes, reactions that needed to be included in the model in order to have metabolic precursors in the network (with no assigned genes), non-enzymatic reactions that have no related gene, and genes described in the annotations but with no assigned function. For an overview of the underlying process, please refer to Fortser et al [32] work on the reconstruction of Saccharomyces cerevisiae metabolic network.

\section{Linear programming for Flux Balance Analysis}

The set of biochemical reactions of the genome-scale metabolic model were formulated as a steady state stoichiometric model:

$$
S \cdot v=0
$$

The details are described elsewhere, for example in Stephanopoulos et al [40]. This model describes cellular behavior under pseudo steady-state conditions, where $S$ is stoichiometric matrix that contains the stoichiometric coefficients corresponding to all internal (balanced) metabolites. $v$ is flux vector that corresponds to the columns of $S$. Given a set of experimentally-driven constraints, former equation was solved by using linear programming, the approach known as flux balance analysis, or FBA [16].

Since the number of reactions is typically larger than the number of metabolites, the system becomes underdetermined. In order to obtain a feasible solution for the intracellular fluxes, an optimization criterion on metabolic balances has to be imposed. This can be formulated by maximizing one of the biochemical reactions, e. g. biomass equation, subject to the mass balance and the capacity constraints.

For instance,

$$
\begin{gathered}
\operatorname{Max}\left(v_{i}\right) \quad \text { subject to } S \cdot v_{j}=0 \quad \forall j \in \mathrm{N} \\
v_{j, \text { irr }} \in \mathrm{R}^{+} \\
v_{j, \text { rev }} \in \mathrm{R} \\
v_{j, \text { const }} \in \mathrm{R}, v_{\text {min }}<v_{j, \text { const }}<v_{\max } \\
v_{j, \text { uptake }} \in \mathrm{R}, v_{\text {min }}<v_{j, \text { uptake }}<v_{\text {max }}
\end{gathered}
$$

where $v_{j}$ is the rate of the $j^{t h}$ reaction. The elements of the flux vector $v$ were constrained for the definition of reversible and irreversible reactions, $v_{j, \text { rev }}$ and $v_{j, \text { irr }}$, respectively. Additionally, two set of equations were established, $v_{j}$, const , constrained metabolic reactions, and $v_{j}$, uptake, uptake reactions, which were bound by experimentally determined values from the literature. Biomass synthesis was considered as a drain of precursors or building blocks into a hypothetical biomass component. Flux through biomass synthesis reaction, being the biomass formation rate, is directly related to growth of the modeled organism [40]. Table 3 shows the biomass composition that was considered in the $i$ Syn 669 metabolic model.

Simulations were performed with the OptGene software [37]. Some capacity constraints had to be added in order to have a feasible solution for the linear programming problem. As an example, maximum uptake rates were determined as follows: maximum glucose uptake rate under heterotrophic conditions was found to be 0.85 mmol glucose $\mathrm{g}_{\mathrm{DW}}{ }^{-1} \mathrm{~h}^{-1}$ [23]. Maximum $\mathrm{CO}_{2}$ uptake rate was found to be $3.7 \mathrm{mmol} \mathrm{CO}_{2} \mathrm{gDw}^{-1} \mathrm{~h}^{-1}$ [24]. Additionally, we fixed the maintenance requirement for the heterotrophic case to be 1.67 ATP moles per mole of glucose consumed as was determined by ref [24], and was maintained for autotrophic and mixotrophic growth.

\section{MOMA algorithm}

Segre et al [17] introduced the method of minimization of metabolic adjustment (MOMA) to better understand the flux states of mutants. MOMA is based on the same stoichiometric constraints as FBA, but relaxes the assumption of optimal growth flux for the mutants, testing the hypothesis that the corresponding flux distribution is better approximated by the flux minimal response to the perturbation than by the optimal one.

MOMA algorithm searches for a point in the feasible space of the solutions space of the knock-out $\left(\Phi^{j}\right)$ that has minimal distance from a given flux vector $w$. The goal is to find the vector $x \in \Phi^{\mathrm{j}}$ such that the Euclidean distance

$$
D(w, x)=\sqrt{\sum_{i=1}^{N}\left(w_{i}-x_{i}\right)^{2}}
$$

is minimized. For details, please address to Segre et al [17].

\section{Reporter Features algorithm}

Reporter Features software [48] works on three kinds of information - network, omics data and association between genes and the nodes in the network. We have used Reporter Features for a transcriptomic analysis, so our three files were $p$-values file, resulting from a Student $\mathrm{t}$-test run on transcriptomic data, interaction file, where reactions are connected to the corresponding substrates and products, and association file, where gene 
are associated to reactions they are involved in, either by coding for the enzyme or by regulating the gene that codes for the enzyme.

In brief, Reporter algorithm converts the p-value for a given node to a $\mathrm{z}$-score by using the inverse normal cumulative distribution function $\left(\mathrm{cdf}^{-1}\right)$.

$$
\mathrm{z}_{\text {gene } \mathrm{i}}=\mathrm{cdf}^{-1}\left(1-\mathrm{p}_{\text {gene i }}\right)
$$

After scoring each non-feature node in this fashion, we need to calculate the score of each feature $j, \mathrm{z}_{\text {feature } j}$. We used the scoring method based on distribution of the means, which is a test for the null hypothesis "genes adjacent to feature $j$ display their normalized average response by chance". In particular, the score of each feature $j$ is defined as the average of the scores of its neighbour $N_{j}$ nodes (genes), i.e.:

$$
z_{\text {feature } j}=\frac{1}{N_{j}} \sum_{k=1}^{N_{j}} z_{\text {gene } k}
$$

To evaluate the significance of each $\mathrm{z}_{\text {feature } j}$, this value should be corrected for the background distribution of $\mathrm{z}$ scores in the data, by subtracting the mean $\left(m_{N}\right)$ and dividing by the standard deviation $\left(s_{N}\right)$ of random aggregates of size $N$.

$$
z_{\text {feature } j}^{\text {correct }}=\frac{\left(z_{\text {feature } j}-m_{N}\right)}{s_{N}}
$$

\section{Additional material}

Additional file 1: iSyn669 reactions to gene connections. Excel file with the list of $i$ Syn669 reactions and its cognate list of genes.

Additional file 2: iSyn669 genome-scale metabolic model in OptGene format. Text file with the stoichiometric model, in OptGene [37] format, with all the constraints needed for its simulation with FBA algorithm.

Additional file 3: Most connected metabolites with filtered cofactors. Supplementary table with most connected metabolites once the cofactors have been filtered.

Additional file 4: iSyn669 metabolic fluxes simulated under four conditions. Excel file with all the reactions simulations and resulting flux ranges from the model simulated under four growth conditions: autotrophy, dark o pure heterotrophy, light-activated heterotrophy and mixotrophy.

Additional file 5: Fluxes of reactions around pyruvate. Flux values (in $\mathrm{mmol} / \mathrm{g} \mathrm{DCW} / \mathrm{h}$ ) for reactions that produce or drain pyruvate in Synechocystis sp. PCC6803 metabolism. Negative sign in bidirectional reactions means pyruvate consumption. Reactions names can be traced in reaction list in Additional files 2 and fluxes can be found in Additional file 4.

Additional file 6: FBA and MOMA simulation values for biomass growth in Synechocystis sp. PCC6803, Escherichia coli and Saccharomyces cerevisiae genome-scale metabolic models. Excel file with the growth values under MOMA simulation for Synechocystis sp. PCC6803, Escherichia coli and Saccharomyces cerevisiae. Data for Synechocystis is original from present work, data for Escherichia coli has been obtained from metabolic model from reference 18 and data for Saccharomyces cerevisiae is from reference 30

Additional file 7: iSyn669 groups of correlated genes in the three sets of arrays of light shift experiments. Word file with the list of iSyn669 correlated genes in "All time points", "Dark to light" and "Light to dark" analyses.

\section{Abbreviations}

BM: biomass; DCW: dry cell weight; FBA: flux balance analysis; MCA: metabolic control analysis; MOMA: minimization of metabolic adjustments; ORF: Open Reading Frame; PEP: phosphoenolpyruvate; ROOM: regulatory onoff minimization of metabolic fluxes; RuBisCO: Ribulose-1,5-bisphosphate carboxylase oxygenase; TCA cycle: tricarboxylic acid cycle

\section{Acknowledgements}

This work was financially supported by MICINN TIN2009-12359 project ArtBioCom, EU FP7-KBBE-2007 project TarPol (contract $n^{\circ} 212894$ ) and EU FP6-NEST-2005 project BioModularH2 (contract nº 043340). AM thanks to Generalitat Valenciana grant BFPI/2007/283 and EN to Ministerio de Educación y Ciencia de España through the program Juan de la Cierva.

\section{Author details}

'Instituto Universitario de Matemática Pura y Aplicada, Universidad Politécnica de Valencia, Camino de Vera 14, 46022 Valencia, Spain. ${ }^{2}$ Departamento de Lenguajes y Ciencias de la Computación, Campus de Teatrinos, Universidad de Málaga, 29071 Málaga, Spain. ${ }^{3}$ Structural and Computational Biology Unit, European Molecular Biology Laboratory, Meyerhofstrasse 1, D-69117 Heidelberg, Germany.

\section{Authors' contributions}

AM and EN conducted the reconstruction and the different analyses. PF and JFU conceived of the study and participated in its design. AM and KRP designed the study and wrote the manuscript. All authors contributed to, read and approved the final manuscript.

Received: 5 February 2010 Accepted: 17 November 2010 Published: 17 November 2010

\section{References}

1. Allen MM, Smith AJ: Nitrogen chlorosis in blue-green algae. Arch Mikrobiol 1969, 69:114-120

2. Tamagnini $P$, Axelsson $R$, Lindberg P, Oxelfelt F, Wunschiers $R$, Lindblad $P$ : Hydrogenases and hydrogen metabolism of cyanobacteria. Microbiol $\mathrm{Mol}$ Biol Rev 2002, 66:1-20, table of contents.

3. Schopf J: The Fossil Record: Tracing the Roots of the Cyanobacterial Lineage. In The ecology of cyanobacteria. Edited by: Whitton B, Potts M. Dordrecht: Kluwer Academic Publishers; 2000:13-35.

4. Shi T, Falkowski PG: Genome evolution in cyanobacteria: the stable core and the variable shell. Proc Natl Acad Sci USA 2008, 105:2510-2515.

5. Tamagnini P, Leitao E, Oliveira P, Ferreira D, Pinto F, Harris DJ, Heidorn T, Lindblad P: Cyanobacterial hydrogenases: diversity, regulation and applications. FEMS Microbiol Rev 2007, 31:692-720.

6. Lindberg P, Park S, Melis A: Engineering a platform for photosynthetic isoprene production in cyanobacteria, using Synechocystis as the model organism. Metab Eng 2010, 12:70-79.

7. Wu GF, Wu QY, Shen ZY: Accumulation of poly-beta-hydroxybutyrate in cyanobacterium Synechocystis sp. PCC6803. Bioresour Technol 2001, 76:85-90.

8. Liu X, Curtiss R: Nickel-inducible lysis system in Synechocystis sp. PCC 6803. Proc Natl Acad Sci USA 2009, 106:21550-21554.

9. Navarro E, Montagud A, Fernández de Córdoba P, Urchueguía JF: Metabolic flux analysis of the hydrogen production potential in Synechocystis sp. PCC6803. Int J Hydrogen Energy 2009, 34:8828-8838.

10. McHugh K: Hydrogen production methods Alexandria, Virginia: MPR Associates, Inc; 2005 
11. Turner J, Sverdrup G, Mann M, Maness P, Kroposki B, Ghirardi M, Evans R, Blake D: Renewable hydrogen production. International Journal Energy Research 2008, 32:379-407.

12. Herrero A, Flores E: The cyanobacteria: molecular biology, genomics, and evolution Norfolk, UK: Caister Academic Press; 2008.

13. Oberhardt MA, Palsson BO, Papin JA: Applications of genome-scale metabolic reconstructions. Mol Syst Biol 2009, 5:320.

14. Patil KR, Akesson M, Nielsen J: Use of genome-scale microbial models for metabolic engineering. Curr Opin Biotechnol 2004, 15:64-69.

15. Varma A, Palsson BO: Metabolic capabilities of Escherichia coli: II. Optimal growth patterns. J Theor Biol 1993, 165:503-522.

16. Edwards J, Ramakrishna R, Schilling C, Palsson B: Metabolic flux balance analysis. In Metabolic engineering. Edited by: Lee S, Papoutsakis E. New York: Marcel Dekker Inc; 1999.

17. Segre D, Vitkup D, Church GM: Analysis of optimality in natural and perturbed metabolic networks. Proc Natl Acad Sci USA 2002, 99:15112-15117.

18. Shlomi T, Berkman O, Ruppin E: Regulatory on/off minimization of metabolic flux changes after genetic perturbations. Proc Natl Acad SCi USA 2005, 102:7695-7700.

19. Rapoport TA, Heinrich R, Jacobasch G, Rapoport S: A linear steady-state treatment of enzymatic chains. A mathematical model of glycolysis of human erythrocytes. Eur J Biochem 1974, 42:107-120.

20. Kacser H, Burns JA: The control of flux. Symp Soc Exp Biol 1973, 27:65-104

21. Kaneko T, Sato S, Kotani H, Tanaka A, Asamizu E, Nakamura Y, Miyajima N, Hirosawa M, Sugiura M, Sasamoto S, et al: Sequence analysis of the genome of the unicellular cyanobacterium Synechocystis sp. strain PCC6803. II. Sequence determination of the entire genome and assignment of potential protein-coding regions (supplement). DNA Res 1996, 3:185-209

22. Kaneko T, Nakamura $Y$, Sasamoto S, Watanabe A, Kohara M, Matsumoto M, Shimpo S, Yamada M, Tabata S: Structural analysis of four large plasmids harboring in a unicellular cyanobacterium, Synechocystis sp. PCC 6803. DNA Res 2003, 10:221-228.

23. Yang C, Hua Q, Shimizu K: Metabolic flux analysis in Synechocystis using isotope distribution from 13C-labeled glucose. Metab Eng 2002, 4:202-216.

24. Shastri AA, Morgan JA: Flux balance analysis of photoautotrophic metabolism. Biotechnol Prog 2005, 21:1617-1626.

25. Fu P: Genome-scale modeling of Synechocystis sp. PCC6803 and prediction of pathway insertion. Journal of Chemical Technology \& Biotechnology 2009, 84:473-483.

26. Karp PD, Ouzounis CA, Moore-Kochlacs C, Goldovsky L, Kaipa P, Ahren D, Tsoka S, Darzentas N, Kunin V, Lopez-Bigas N: Expansion of the BioCyc collection of pathway/genome databases to 160 genomes. Nucleic Acids Res 2005, 33:6083-6089.

27. Kanehisa M, Araki M, Goto S, Hattori M, Hirakawa M, Itoh M, Katayama T, Kawashima S, Okuda S, Tokimatsu T, Yamanishi Y: KEGG for linking genomes to life and the environment. Nucleic Acids Res 2008, 36: D480-484.

28. Chang A, Scheer M, Grote A, Schomburg I, Schomburg D: BRENDA, AMENDA and FRENDA the enzyme information system: new content and tools in 2009. Nucleic Acids Res 2009, 37:D588-592.

29. The universal protein resource (UniProt). Nucleic Acids Res 2008, 36: D190-195.

30. Weise S, Grosse I, Klukas C, Koschutzki D, Scholz U, Schreiber F, Junker BH: Meta-All: a system for managing metabolic pathway information. BMC Bioinformatics 2006, 7:465.

31. Feist AM, Herrgard MJ, Thiele I, Reed JL, Palsson BO: Reconstruction of biochemical networks in microorganisms. Nat Rev Microbiol 2009, 7:129-143

32. Forster J, Famili I, Fu P, Palsson BO, Nielsen J: Genome-scale reconstruction of the Saccharomyces cerevisiae metabolic network. Genome Res 2003, 13:244-253.

33. Karp PD, Paley S, Romero P: The Pathway Tools software. Bioinformatics 2002, 18(Suppl 1):S225-232.

34. Bairoch A: The ENZYME database in 2000. Nucleic Acids Res 2000 28:304-305.

35. Yang C, Hua Q, Shimizu K: Quantitative analysis of intracellular metabolic fluxes using GC-MS and two-dimensional NMR spectroscopy. J Biosci Bioeng 2002, 93:78-87.
36. Pearce J, Carr NG: The metabolism of acetate by the blue-green algae, Anabaena variabilis and Anacystis nidulans. J Gen Microbiol 1967, 49:301-313.

37. Patil KR, Rocha I, Forster J, Nielsen J: Evolutionary programming as a platform for in silico metabolic engineering. BMC Bioinformatics 2005, 6:308

38. Feist AM, Henry CS, Reed JL, Krummenacker M, Joyce AR, Karp PD, Broadbelt $L$, Hatzimanikatis V, Palsson BO: A genome-scale metabolic reconstruction for Escherichia coli K-12 MG1655 that accounts for 1260 ORFs and thermodynamic information. Mol Syst Biol 2007, 3:121.

39. Zelezniak A, Pers TH, Soares S, Patti ME, Patil KR: Metabolic network topology reveals transcriptional regulatory signatures of type 2 diabetes. PLoS Comput Biol 2010, 6:e1000729.

40. Stephanopoulos G, Aristidou AA, Nielsen JH: Metabolic engineering: principles and methodologies San Diego: Academic Press; 1998.

41. Schuetz R, Kuepfer L, Sauer U: Systematic evaluation of objective functions for predicting intracellular fluxes in Escherichia coli. Mol Syst Biol 2007, 3:119.

42. Anderson SL, McIntosh L: Light-activated heterotrophic growth of the cyanobacterium Synechocystis sp. strain PCC 6803: a blue-light-requiring process. J Bacteriol 1991, 173:2761-2767.

43. Carr NG, Whitton BA: The Biology of cyanobacteria Berkeley: University of California Press; 1982

44. Pelroy RA, Rippka R, Stanier RY: Metabolism of glucose by unicellular blue-green algae. Arch Mikrobiol 1972, 87:303-322.

45. Loferer-Krossbacher M, Klima J, Psenner R: Determination of bacterial cell dry mass by transmission electron microscopy and densitometric image analysis. Appl Environ Microbiol 1998, 64:688-694

46. Lawrence BA, Suarez C, DePina A, Click E, Kolodny NH, Allen MM: Two internal pools of soluble polyphosphate in the cyanobacterium Synechocystis sp. strain PCC 6308: an in vivo 31P NMR spectroscopic study. Arch Microbiol 1998, 169:195-200

47. Stephanopoulos G, Alper H, Moxley J: Exploiting biological complexity for strain improvement through systems biology. Nat Biotechnol 2004, 22:1261-1267.

48. Oliveira AP, Patil KR, Nielsen J: Architecture of transcriptional regulatory circuits is knitted over the topology of bio-molecular interaction networks. BMC Syst Biol 2008, 2:17.

49. Patil KR, Nielsen J: Uncovering transcriptional regulation of metabolism by using metabolic network topology. Proc Natl Acad Sci USA 2005, 102:2685-2689.

50. Gill RT, Katsoulakis E, Schmitt W, Taroncher-Oldenburg G, Misra J, Stephanopoulos G: Genome-wide dynamic transcriptional profiling of the light-to-dark transition in Synechocystis sp. strain PCC 6803. J Bacteriol 2002, 184:3671-3681.

51. NCBI Entrez Genome for Synechocystis sp. PCC6803. [http://www.ncbi. nlm.nih.gov/sites/entrez?Db=genome\&Cmd=ShowDetailView\&TermToSearch $=112]$.

52. Pearce J, Leach CK, Carr NG: The incomplete tricarboxylic acid cycle in the blue-green alga Anabaena variabilis. J Gen Microbiol 1969, 55:371-378.

53. Vazquez-Bermudez MF, Herrero A, Flores E: Uptake of 2-oxoglutarate in Synechococcus strains transformed with the Escherichia coli kgtP gene. J Bacteriol 2000, 182:211-215.

54. Peschek GA, Löffelhardt W, Schmetterer G: The phototrophic prokaryotes New York: Kluwer Academic/Plenum; 1999.

55. Rubio FC, Camacho FG, Sevilla JM, Chisti Y, Grima EM: A mechanistic model of photosynthesis in microalgae. Biotechnol Bioeng 2003, 81:459-473.

56. Albertsson P: A quantitative model of the domain structure of the photosynthetic membrane. Trends Plant Sci 2001, 6:349-358.

57. Allen J: Photosynthesis of ATP-electrons, proton pumps, rotors, and poise. Cell 2002, 110:273-276

58. Herdman M, Janvier M, Waterbury J, Rippka R, Stanier R: Deoxyribonucleic Acid Base Composition of Cyanobacteria. Journal of General Microbiology 1979, 111:63-71.

59. Tasaka Y, Gombos Z, Nishiyama Y, Mohanty P, Ohba T, Ohki K, Murata N: Targeted mutagenesis of acyl-lipid desaturases in Synechocystis: evidence for the important roles of polyunsaturated membrane lipids in growth, respiration and photosynthesis. EMBO J 1996, 15:391-396. 
60. Miao X, Wu Q, Wu G, Zhao N: Changes in photosynthesis and pigmentation in an agp deletion mutant of the cyanobacterium Synechocystis sp. Biotechnol Lett 2003, 25:391-396.

61. Burrows EH, Chaplen FW, Ely RL: Optimization of media nutrient composition for increased photofermentative hydrogen production by Synechocystis sp. PCC6803. International Journal of Hydrogen Energy 2008 33:6092-6099

doi:10.1186/1752-0509-4-156

Cite this article as: Montagud et al: Reconstruction and analysis of genome-scale metabolic model of a photosynthetic bacterium. BMC Systems Biology 2010 4:156.

Submit your next manuscript to BioMed Central and take full advantage of:

- Convenient online submission

- Thorough peer review

- No space constraints or color figure charges

- Immediate publication on acceptance

- Inclusion in PubMed, CAS, Scopus and Google Scholar

- Research which is freely available for redistribution

Submit your manuscript at www.biomedcentral.com/submit
C Biomed Central 